\title{
Increasing routine cancer screening among underserved populations through effective communication strategies: Application of a health literacy framework
}

\author{
Alicia L. Best, PhD, MPH, \\ Department of Community and Family Health, College of Public Health, University of South \\ Florida, 13201 Bruce B. Downs Blvd, Tampa, Florida 33612, abest@health.usf.edu, Telephone: \\ 813-974-5290, Fax: 813-974-5172 \\ Cheryl Vamos, PhD, MPH, \\ Department of Community and Family Health, College of Public Health, University of South \\ Florida, 13201 Bruce B. Downs Blvd, Tampa, Florida 33612

\section{Seul Ki Choi, PhD, MPH,} \\ Department of Health Promotion, Education, and Behavior, Arnold School of Public Health, \\ University of South Carolina, 915 Greene Street, Columbia, South Carolina 29208

\section{Erika L. Thompson, PhD, MPH,} \\ Department of Community and Family Health, College of Public Health, University of South \\ Florida, 13201 Bruce B. Downs Blvd, Tampa, Florida 33612 \\ Ellen Daley, PhD, MPH, and \\ Department of Community and Family Health, College of Public Health, University of South \\ Florida, 13201 Bruce B. Downs Blvd, Tampa, Florida 33612

\section{Daniela B. Friedman, PhD} \\ Department of Health Promotion, Education, and Behavior, Arnold School of Public Health, \\ University of South Carolina, 915 Greene Street, Columbia, South Carolina 29208
}

\section{Abstract}

\footnotetext{
Cancer prevention and control efforts serve as national priorities, as cancer is the second leading cause of death in the United States. In addition, cancer disparities exist, with racial/ethnic minority, low-income, and uninsured populations suffering the greatest burden. The goal of this paper is to describe the role that effective health communication can play in increasing routine cancer screening among medically underserved populations, thus decreasing persistent health disparities. For this paper, we applied Sorenson's integrated model of health literacy as a framework for identifying communication gaps and opportunities that can help improve cancer screening specifically at federally qualified health centers (FQHCs). This integrated model consists of four interrelated dimensions: access, understand, appraise, and apply. Employing communication strategies across this health literacy framework has the potential to facilitate
}

Corresponding Author: Alicia L. Best PhD, MPH,Department of Community and Family Health, College of Public Health, University of South Florida, 13201 Bruce B. Downs Blvd, Tampa, Florida 33612, abest@ health.usf.edu, Telephone: 813-974-5290, Fax: 813-974-5172. 
improved decision making and cancer screening outcomes among the most underserved populations.

Routine cancer screening such as mammography, colonoscopy, and Papanicolaou (Pap) testing are highly prioritized to address cancer disparities as they have the potential to greatly reduce both incidence and mortality rates [1]. Despite this increased priority, racial/ ethnic minority and low-income populations continue to underutilize routine screening, and consequently are more likely to be diagnosed with later stage disease compared to nonHispanic White and higher socioeconomic status populations [2]. In addition, disparities in cancer prevention and early detection lead to poorer outcomes and lower survival after cancer diagnosis among racial/ethnic minority and low-income populations compared to their counterparts [3]. Efforts to increase cancer screening rates for these vulnerable populations have been made [4]; yet, disparities persist. The goal of this paper is to describe the role that effective health communication within a health literacy framework can play in increasing routine cancer screening among medically underserved populations.

Federally qualified health centers (FQHCs) are funded by the Health Resources and Services Administration to provide preventive and primary health care services, including cancer screening, to the nation's most vulnerable populations [5]. FQHCs offer health care on income-based sliding fee scales, are required to accept all patients regardless of their ability to pay for services, and are strategically located in medically underserved communities [5]. These characteristics position FQHCs as invaluable partners in improving routine cancer screening among the medically underserved. However, many underserved populations still use hospital emergency departments for primary care needs [6]. In 2014, the up-to-date cancer screening rate among $\mathrm{FQHC}$ patients was $56.3 \%$ and $34.5 \%$ for cervical and colorectal cancer, respectively [7]; while the national average of up-to-date screening was $83.0 \%$ and over $50 \%$ for cervical and colorectal cancer, respectively, during the same period [8]. This could be due, in part, to lack of awareness and/or understanding of the FQHC's role in alleviating the cost concern among uninsured populations [9].

Health communication is an interdisciplinary, applied research field focused on "the production and exchange of information to inform, influence or motivate individual, institutional and public audiences about health issues" (p. 4) [10]. Health communication provides important strategies for reaching diverse populations with health information and services. A goal outlined in Healthy People 2020 calls for improving health literacy by the use of health communication approaches to achieve health equity [1]. Additionally, Thiede and McIntyre [11] argue that "information is the essential prerequisite for access" (p. 1169) to health services, and that health communication must be emphasized as a means of addressing health inequity.

Health literacy requires "people's knowledge, motivation and competences to access, understand, appraise, and apply health information in order to make judgments and take decisions in everyday life concerning healthcare, disease prevention and health promotion to maintain or improve quality of life during the life course" [12]. The significant impact of health literacy on population health has been well established as poor health literacy has been associated with a range of adverse health behaviors, inadequate use of health care 
services, and negative health outcomes [13]. Although the association of poor health literacy on health disparities has been established, the specific mechanisms linking these two require more research [14]. Nonetheless, health literacy is an evolving, multidimensional concept that highlights the critical competencies, skills and abilities needed among individuals and systems to improve health across different contexts.

Sorensen and colleagues [12] propose an integrated model of health literacy across four dimensions. Each of the four dimensions is interrelated and builds upon the previous, starting with access, which "refers to the ability to seek, find and obtain health information" (p. 9) [12]. The second dimension involves the ability to understand or comprehend information once it has been accessed. The third dimension is appraise, which is one's "ability to interpret, filter, judge and evaluate the health information that has been accessed" (p. 9) [12]. The final dimension is apply, which refers to the "ability to communicate and use" health information for informed health and healthcare decision-making (p. 9) [12]. Understanding the current health communication gaps and opportunities related to routine cancer screening among medically underserved populations at each health literacy dimension could facilitate access to and utilization of services, ultimately addressing the persistent health disparities among these populations. Thus, we propose the use of health communication methods and techniques across health literacy dimensions to address routine cancer screening among underserved populations.

\section{Access}

Enabling access to cancer screening information and services is a foundational component to eliminating cancer disparities, especially among medically underserved populations. Access to care is a multidimensional construct that involves factors which either "facilitate or impede the opportunity to use health services." (p. 1169) [11]. Access to cancer screening involves how, where, when and to whom information and services are made available. FQHCs bring preventive and primary health care to medically underserved communities, covering only one aspect of access - availability. However, access cannot be fully granted if communities are not aware of resources; thus, health communication strategies are needed to enhance the visibility of FQHCs among target communities. There is a lack of research related to how FQHCs are marketed to target communities and whether or not current marketing approaches are effective. More importantly, there is insufficient literature to answer the questions: 1) What do medically underserved populations know about FQHCs and how do they perceive them?; and 2) How are medically underserved populations currently accessing information on cancer screening?

\section{Understand}

Even when populations are aware of available cancer screening services and programs, there are various factors that impact their understanding of those services which could impede usage. Almufleh and colleagues [15] conducted a study in which individuals residing in medically underserved areas were screened for cardiovascular risk factors, and when abnormal results were detected, individuals who were uninsured and lacked a primary care provider were referred to FQHCs. Findings indicated that of the nearly 1,900 patients 
referred to FQHCs for follow-up care, only $11 \%$ actually sought treatment [15]. The majority of these patients cited lack of health insurance and inability to afford services as the main reason for not adhering to follow-up recommendations [15]. This raises the following research question: To what extent do people understand the payment structure of FQHCs (i.e., sliding fee scale)? There is a lack of research focused on the level of understanding of the FQHC model among underserved communities.

Additionally, the complexity and variability of cancer screening guidelines also clouds understanding and may negatively impact screening rates among underserved populations. For example, the United States Preventive Services Task Force (USPSTF) recently amended their breast cancer screening guidelines, increasing the age for which mammography is recommended [16]. Similarly, the USPSTF amended guidelines for Pap testing to include the recommendation of screening every five years if combined with human papillomavirus (HPV) testing for women 30-65 [17]. Identifying ways to best to communicate cancer prevention and screening guidelines based on evolving science will remain a health communication challenge in public health. Conducting formative research with potential FQHC users to develop and test effective and relevant communication strategies will be essential to assure that underserved populations understand how and why cancer screening services can be beneficial to them [18]. This includes facilitating the procedural knowledge among the target population to assure that they understand how each procedure works, such as pertinent follow-up care instructions. For example, previous research has found substantial gaps in procedural knowledge regarding the Pap test, where this test was confused with other sexual and reproductive health screenings [19]. Thus, in communicating both general health and patient specific information, it is essential that all written materials abide by the plain language principle where information is easy to read and understand [20]. Such principles include using common and everyday words, active voice, and short sentences [21]. Other health literacy techniques such as AskMe3 [22] could also be better utilized where patients are empowered to have pre-identified questions prepared to ask their provider, ultimately fostering patient-provider communication.

\section{Appraise}

When cancer screening information and services are made available in an understandable manner to underserved communities, the next layer of health literacy is an individual's ability to evaluate the applicability of the information and services to their specific needs. Two important health communication strategies to assist with making information more acceptable to various populations include message targeting and tailoring. Although often used interchangeably, there is an important distinction between targeting and tailoring in health communication. Targeting focuses on characteristics of a group, whereas tailoring is focused on individual characteristics [23]. With regard to cancer screening among underserved populations, targeted health communication efforts have addressed cultural beliefs around cancer [24], racial/ethnic variability in cancer burden [25], and engaging patients in informed choice and discussions about cancer screening [26]. Tailored health communication interventions have focused on delivering cancer screening recommendations based on individual characteristics such as personal barriers to screening, family history, and genetics [27]. In addition, decision support systems and tools have become increasingly 
popular and hold great promise in identifying the advantages and disadvantages of engaging in various health screening and treatment regimens and can effectively present information to end users in a culturally and linguistically appropriate manner while considering a patient's unique medical history and personal concerns [26].

\section{Apply}

The ability to communicate with healthcare providers and family members about cancer risk and appropriate screening, and actually utilize screening services is the final health literacy dimension described by Sorensen and colleagues [12]. As identified in the appraise dimension above, decision aids could help patients in their informed decision-making - one of the key outcomes of a health literate individual - by helping them weigh advantages and disadvantages regarding screening in a patient-centered manner. Moreover, strategies to assist patients in disclosing their cancer diagnosis to family members should be readily available as such a diagnosis could be perceived as stigmatizing or culturally taboo in some populations and further contribute to lack of cancer screening and early detection [28]. Furthermore, ensuring individuals both understand and act on the prescribed follow-up care and that they are not lost throughout the various processes of the healthcare system should remain a priority in addressing the persistent cancer disparities. To this end, the use of health navigators in employing patient-centered and culturally competent health communication interventions serve as one example to facilitate an individual's ability to engage in health behaviors across the cancer continuum.

\section{Reflections and Recommendations}

This paper applies Sorenson's integrated model of health literacy as a framework for identifying communication gaps and opportunities to help improve cancer screening among medically underserved populations. Specifically, health communication techniques can be employed across the four health literacy dimensions (access, understand, appraise, apply), ultimately facilitating underserved populations in their ability to be engaged and empowered in shared decision-making and patient-centered care. Although presented as a continuum, the health literacy dimensions are not necessarily mutually exclusive, nor linear, and as such, strategies for improving health literacy may be relevant across multiple dimensions. For example, decision aids can be used to help individuals understand, appraise, and apply health information to make screening decisions.

When identifying communication gaps and opportunities through a health literacy framework, an important consideration when raising awareness and understanding of screening services through $\mathrm{FQHCs}$ is to ensure that the system is able to keep up with increasing demand. FQHCs must be equipped with resources (staff, personnel, equipment, labs/technology, etc.) to provide quality screening services. Literature indicates that FQHCs face challenges to providing screening services to their patients due to workload, scarce resources, lack of incentives, and lack of referral sources [29]. Not all FQHCs have the capacity to provide cancer screening, especially those that require special equipment or skills such as mammography and colonoscopy. FQHCs use various strategies to provide their patients with specialty services, including leveraging personal relationships with 
specialists for patient referrals, contracting with community hospitals, using telecommunications, or partnering with local health systems (e.g. government or safety-net hospital) that provides access to all subspecialties [30].

Future research and practice could assess and ensure that FQHCs follow the 10 attributes of a health literate organization [31]. This resource is an invaluable blueprint where organizations can assess gaps and opportunities to improve their health literacy efforts across system levels. While a health literate organization aims to integrate health literacy into their mission and planning, a critical component is utilizing health literacy strategies for communication with clients [31]. A health literate organization must also effectively interact with other stakeholders and systems (e.g., Medicaid enrollment, social services, WIC offices, etc.) to leverage and improve the coordination of patient care [32]. Thus, health communication and health literacy efforts are essential in promoting health literate organizations. Establishing health literacy efforts in FQHCs through facilitation of patient navigation and informed decision-making can address cancer screening concerns among medically underserved populations.

In addition to system-level interventions, patient-level efforts must also be integrated to promote access to and uptake of cancer screenings. Thus, a community-engaged approach will be beneficial to identify target populations that are at risk for cancer and/or medically underserved, develop and test appropriate health communication materials regarding both the role of FQHCs and the topic of cancer, and sustain interventions/programs in the community, while assuring that information and services are culturally and linguistically appropriate [33].

In conclusion, utilizing health communication techniques to facilitate access, understanding, appraisal and use of cancer screening information and services among underserved populations serve as national priorities. FQHCs provide an important venue to facilitate cancer screening among this population; thus, better communication approaches are needed to increase their visibility and utilization. These efforts are of public health significance and can assist in promoting uptake of cancer screening modalities and early identification of cancer, and ultimately reducing persistent cancer-related health disparities.

\section{Acknowledgements:}

Friedman is partially supported by the South Carolina Cancer Prevention and Control Research Network under Cooperative Agreement Number U48/DP005000-01S2 from the Centers for Disease Control and Prevention (Prevention Research Centers) and the National Cancer Institute. Best is an affiliate of the Cancer Prevention and Control Research Network. The content is solely the responsibility of the authors and does not necessarily represent the official views of the National Cancer Institute or Centers for Disease Control and Prevention.

\section{References}

1. US Department of Health and Human Services, and Office of Disease Prevention and Health Promotion. 2010 Healthy people 2020. https://www.healthypeople.gov/2020/. Accessed 23 May 2016.

2. National Cancer Institute. 2008 Cancer health disparities fact sheet. http://www.cancer.gov/aboutnci/organization/crchd/cancer-health-disparities-fact-sheet. Accessed 10 October 2016. 
3. Yu Xue Qin. 2009 Socioeconomic disparities in breast cancer survival: relation to stage at diagnosis, treatment and race. BMC Cancer 9:364. doi:10.1186/1471-2407-9-364 [PubMed: 19828016]

4. Centers for Disease Control and Prevention. National breast and cervical cancer early detection program (NBCCEDP). https://www.cdc.gov/cancer/nbccedp/. Accessed 7 July 2016.

5. Health Resources and Services Administration. What are Federally Qualified Health Centers (FQHCs)? http://www.hrsa.gov/healthit/toolbox/RuralHealthITtoolbox/Introduction/qualified.html. Accessed 23 May 2016.

6. Agarwal Parul, Bias Thomas K, Vasile Emily, Moore Louise, Davis Stephen, and Davidov Danielle. 2015 Exploring health insurance status and emergency department utilization. Health Serv Res Manag Epidemiol 2:2333392815606094. [PubMed: 28462264]

7. Health Resources and Service Administration. 2014 Health Center Data. http://bphc.hrsa.gov/uds/ datacenter.aspx. Accessed May 132016.

8. American Cancer Society. 2014 Cancer Prevention \& Early Detection Facts \& Figures 2014. Atlanta: American Cancer Society.

9. Best AL, Strane A, Christie O, Bynum SA, Wiltshire J In Press. Examining the influence of cost concern and awareness of low cost health care on cancer screening among the medically underserved. J Health Care Poor Underserved.

10. Maibach Edward W, Abroms Lorien C, and Marosits Mark. 2007 Communication and marketing as tools to cultivate the public's health: a proposed "people and places" framework. BMC Public Health 7:88. [PubMed: 17519027]

11. Thiede Michael, and Di McIntyre. 2008 Information, communication and equitable access to health care: a conceptual note. Cad Saude Publica 24 (5):1168-1173. [PubMed: 18461247]

12. Sørensen Kristine, Van den Broucke Stephan, Fullam James, Doyle Gerardine, Pelikan Jürgen, Slonska Zofia, and Brand Helmut. 2012 Health literacy and public health: a systematic review and integration of definitions and models. BMC Public Health 12:80. [PubMed: 22276600]

13. Berkman Nancy D, Sheridan Stacey L, Donahue Katrina E, Halpern David J, and Crotty Karen. 2011 Low health literacy and health outcomes: an updated systematic review. Ann Intern Med 155 (2):97-107 [PubMed: 21768583]

14. Mantwill Sarah, Monestel-Umaña Silvia, and Schulz Peter J. 2015 The relationship between health literacy and health disparities: A systematic review. PLoS One 10 (12):e0145455 [PubMed: 26698310]

15. Almufleh Aws, Gabriel Tori, Tokayer Laura, Comerford Mary, Alaqeel Ahmed, and Kurlansky Paul. 2015 Role of community health outreach program "living for health" ${ }^{\circledR}$ in improving access to federally qualified health centers in Miami-dade county, Florida: a cross-sectional study. BMC Health Serv Res 15:181. [PubMed: 25928016]

16. US Preventive Services Task Force. 2009 Screening for breast cancer: US Preventive Services Task Force recommendation statement. Ann Intern Med 151 (10):716-726. doi: 10.7326/0003-4819-151-10-200911170-00008 [PubMed: 19920272]

17. Moyer Virginia A. 2012 Screening for cervical cancer: US Preventive Services Task Force recommendation statement. Ann Intern Med 156 (12):880-891 [PubMed: 22711081]

18. Friedman, Daniela B, Hooker Steven P, Wilcox Sara, Burroughs Ericka L, and Rheaume Carol E. 2012 African American men's perspectives on promoting physical activity:"We're not that difficult to figure out!". J Health Commun 17 (10):1151-1170. [PubMed: 22808914]

19. Daley Ellen, Perrin Kay, Vamos Cheryl, Hernandez Natalie, Anstey Erica, Baker Elizabeth, Kolar Stephanie, and Ebbert Judith. 2013 Confusion about Pap smears: lack of knowledge among highrisk women. J Womens Health 22 (1):67-74.

20. Stableford Sue, and Mettger Wendy. 2007 Plain language: A strategic response to the health literacy challenge. J Public Health Policy 28 (1):71-93 [PubMed: 17363939]

21. Plain Language Action and Information Network. What is plain language? http:// www.plainlanguage.gov/whatisPL/. Accessed 6 December 2016.

22. National Patient Safety Foundation. Ask Me 3: Good questions for your good health. http:// www.npsf.org/?page=askme3. Accessed 7 July 2016.

23. Kreuter Matthew W, and Skinner Celette Sugg. 2000 Tailoring: what's in a name? Health Educ Res 15 (1):1-4. [PubMed: 10788196] 
24. Wang Judy Huei-yu, Marc D Schwartz George Luta, Maxwell Annette E, and Mandelblatt Jeanne S. 2012 Intervention tailoring for Chinese American women: comparing the effects of two videos on knowledge, attitudes and intentions to obtain a mammogram. Health Educ Res 27 (3):523-536. [PubMed: 22327806]

25. Shirazi Mehra, Kimberly K Engelman Olive Mbah, Shirazi Aida, Robbins Irma, Bowie Janice, Popal Rona, Wahwasuck Arlene, Whalen-White Denice, and Greiner Allen. 2015 Targeting and tailoring health communications in breast screening interventions. Prog Community Health Partnersh 9 (2):83-89

26. Smith Sian K, Trevena Lyndal, Simpson Judy M, Barratt Alexandra, Nutbeam Don, and McCaffery Kirsten J. 2010 A decision aid to support informed choices about bowel cancer screening among adults with low education: randomised controlled trial. BMJ 341:c5370. [PubMed: 20978060]

27. Manne Sharon L, Coups Elliot J, Markowitz Arnold, Meropol Neal J, Haller Daniel, Jacobsen Paul B, Jandorf Lina, Peterson Susan K, Lesko Samuel, and Pilipshen Steven. 2009 A randomized trial of generic versus tailored interventions to increase colorectal cancer screening among intermediate risk siblings. Ann Behav Med 37 (2):207-217. [PubMed: 19418107]

28. Webber Colleen, Jiang Li, Grunfeld Eva, and Groome Patti A. In Press. Identifying predictors of delayed diagnoses in symptomatic breast cancer: a scoping review. Eur J Cancer Care.

29. Coronado Gloria D, Petrik Amanda F, Spofford Mark, Talbot Jocelyn, Hoai Do Huyen, and Taylor Victoria M. 2015 Clinical perspectives on colorectal cancer screening at Latino-serving federally qualified health centers. Health Educ Behav 42 (1):26-31. [PubMed: 24952378]

30. Neuhausen Katherine, Grumbach Kevin, Bazemore Andrew, and Phillips Robert L. 2012 Integrating community health centers into organized delivery systems can improve access to subspecialty care. Health Aff 31 (8):1708-1716.

31. Brach Cindy, Keller Debra, Hernandez Lyla M, Baur Cynthia, Dreyer Benard, Schyve Paul, Lemerise Andrew J, and Schillinger Dean. 2012 Ten attributes of health literate health care organizations. Washington, DC: Institute of Medicine of the National Academies.

32. Hernandez Lyla M.How can health care organizations become more health literate?: Workshop summary; National Academies Press; 2012.

33. Jackson Dawnyea D, Owens Otis L, Friedman Daniela B, and Dubose-Morris Ragan. 2015 Innovative and community-guided evaluation and dissemination of a prostate cancer education program for African-American men and women. J Cancer Educ 30 (4):779-785. [PubMed: 25510370] 


\section{Access}

- Increase visibility of FQHCs among target

- Disseminate information in a variety of formats (e.g. print, audio, video, email, mobile, etc.)

- Use various distribution channels (e.g. church bulletin, grocery stores, city buses, mass media, social media, etc.)

- Improve capacity of FQHCs to provide screening

\section{Understand}

- Provide information

on:

- Fee structure at FQHCS

- Procedure of cancer screenings

- Variable cancer screening guidelines

- Make information easy to understand

screening

Figure 1.

Communication opportunities across health literacy dimensions to help improve cancer screening at federally qualified health centers 